\title{
Matices del abordaje endonasal endoscópico extendido en la exéresis de los craneofaringiomas
}

\author{
Marlon Ortiz Machín MD1, Omar López Arbolay PhD1, Peggys O Cruz Pérez MD. ${ }^{1}$ \\ 1 Hospital Clínico Quirúrgico "Hermanos Ameijeiras". San Lázaro \# 701 Centro Habana. La Habana. Cuba.
}

Rev. Chil. Neurocirugía 43: 128-133, 2017

\section{Resumen}

Introducción: Los Craneofaringiomas son tumores embrionarios que se originan en la parte anterosuperior de la hipófisis, con un frecuente crecimiento supraselar. El crecimiento supraselar puede involucrar o no el hipotálamo anterior, medio o posterior en dependencia del sentido del vector de crecimiento anteroposterior. Método: Se operaron 40 pacientes en el Hospital Hermanos Ameijeiras donde se realizó un abordaje endonasal endoscópico extendido transplanum transtuberculum transmedio selar; trans-selar o trans-doso selar, en dependencia de la clasificación de Kassam; se determinó el compartamiento clínico pre y postoperatorio aplicando la clasificación de Elliot y colaboradores. Resultados: Se observó una mejoría del estatus visual, no así del estatus pituitario ni hipotalámico, sin embargo, se logró una estabilización de los mismos. El acceso quirúrgico y el grado de resección tumoral fueron excelentes, con resección total en el 92,5\% y la complicación más frecuente fue la diabetes insípida.

Palabras clave: Craneofaringioma, abordaje endonasal endoscópico, tubérculo selar, plano esfenoidal.

\begin{abstract}
Introduction: Craneopharyngiomas are embryonic tumors located in anterosuperior part of the hypophysial gland with frecuent enlargement to supraselar area. It can involved the anterior, medium o posterior hipothalamus zone depended of the growing vector of the tumors. Method: 40 patient was operated in "Hermanos Ameijeiras" Hospital, the type of extended endonasal endoscopic approach was performed depended on Kassam classification, and the clinical status was assessed according Elliot classification. Outcome: Visual status improved after surgery, however pituitary and hypothalamic status persisted, but was stabilized. Surgical approach was excellent, and gross total resection was achieved in $92,5 \%$. The most frecuent complication was insipid diabetes.
\end{abstract}

Key words: Craneopharyngioma, endonasal endoscopic approach, selar tubercle, esfenoidal planum.

\section{Introducción}

Los Craneofaringiomas son tumores embrionarios que se originan en la parte anterosuperior de la adenohipófisis, con un crecimiento mayormente supraselar y en pequeño porciento intraselar $^{1}$. El crecimiento supraselar puede involucrar o no el hipotálamo anterior, medio o posterior en dependencia del sentido del vector de crecimiento an- teroposterior. Kassam, en dependencia de la localización del componente sólido lo clasifica en Preinfundibular, Infundibular y Retroinfundibular, así como los que crecen libremente en el tercer ventrículo². Pugget en dependencia del crecimiento caudo-rostral los clasifica en grado 0: no alcanza el hipotálamo; grado 1: contacta el hipotálamo y lo desplaza pero no lo infiltra y grado 2: involucra e infiltra el hipotá- lamo ${ }^{3}$. La unificación de ambas clasificaciones nos permiten identificar, planificar e incluso predecir cual sería el abordaje endoscópico ideal para cada caso. En estudios anteriores se ha enfatizado que la parte sólida tumoral es la de mayor importancia desde el punto de vista fisiopatogénico y es donde debe ser dirigido fundamentalmente la diana quirúrgica, sin despreciar dentro del mismo abordaje la evacuación de 
la parte quística la cual ejerce un fenómeo compresivo sobre las estructuras subyacentes ${ }^{4}$.

\section{Material y Método}

Independientemente de la localización tumoral en el área supraselar el abordaje debe ser de inicio: TransplanumTranstuberculum-Transmedio-selar (Figura 1).

Este abordaje endoscópico establece una osteotomía trapezoidal con base mayor superior, limitado lateralmente por los recesos óptico-carotídeos mediales (punto Key Hole) y expandido superiormente a nivel del plano esfenoidal. Este abordaje permite exponer los tres complejos anatómicos esenciales relacionados con el tumor, los cuales tienen una composición anatomofuncional independiente:

1. Complejo inferior Tubero-hipofisario.

2. Complejo medio Quiasmático.

3. Complejo superior Cerebral anterior. Una vez identificada la proyección de los tres complejos anatómicos se procede a realizar la apertura dural la cual debe ser realizada entre la proyección del complejo inferior y medio (área infraquiasmática) pues en esta área existe un espacio que no contacta directamente con las estructuras neurovasculares, a diferencia del extremo superior donde el quiasma óptico esta desplazado ventralmente por el tumor así como en el extremo inferior donde se encuentra el seno coronal y la glándula hipófisis (Figura 2) La apertura dural se extiende inferiormente previa coagulación del seno coronal hasta exponer la cara anterosuperior de la hipófisis. Posteriormente, de forma cuidadosa se realiza la extensión superior de la incisión sin dañar el quiasma óptico y la arteria cerebral anterior. Seguidamente se realiza la incisión horizontal completando así la incisión cruciforme.

Antes de realizar la identificación y disección tumoral debe realizarse una apertura del diafragma selar con el objetivo de liberar el tallo hipofisario, lo cual favorecería a la movilización de la glándula en caso de ser necesario sin daños por cizallamiento. Es importante identificar precozmente la red vascular circuninfundibular la cual garantiza la conexión funcional entre el hipotálamo y la adenohipósis (Figura 3).

Posteriormente, se identifican 2 corredores quirúrgicos: el infraquiasmático y

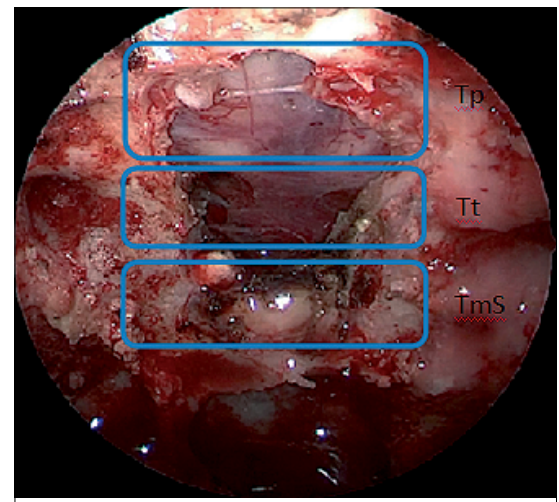

Figura 1. Abordaje Endonasal Endoscópico Transplanum-transtuberculum-transmedio selar. Tp: Transplanum, Tt: Transtuberculum; TmS: Transmedio selar.

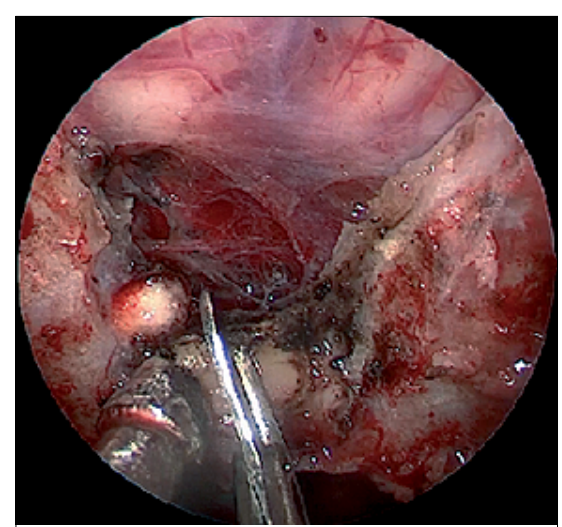

Figura 3. Apertura del diafragma selar hasta liberar el tallo hipofisario, lo cual permite movilizar la glándula hipofisaria.

el supraquiasmático. De forma general el corredor infraquiasmático es el fundamental, el cual frecuentemente se encuentra expandido por el tumor (Figura 4).

Posteriormente, se determina si el tumor es preinfundibular, infundibular o retroinfundibular. En el caso de ser Preinfundibular, no es necesario extender la osteotomía. En los Craneofaringiomas infundibulares en ocasiones es necesario extender la osteotomía inferiormente hasta exponer toda la glándula con el objetivo de tener más visualización caudo-rostral. Es importante en estos casos realizar la incisión en el tallo hipofisario de forma longitudinal a la hora de realizar la disección del tumor, con el objetivo de preservar la mayor cantidad de fibras nerviosas posibles del tallo hipofisario, aunque la diabetes insípida es la regla. En los craneofarin-

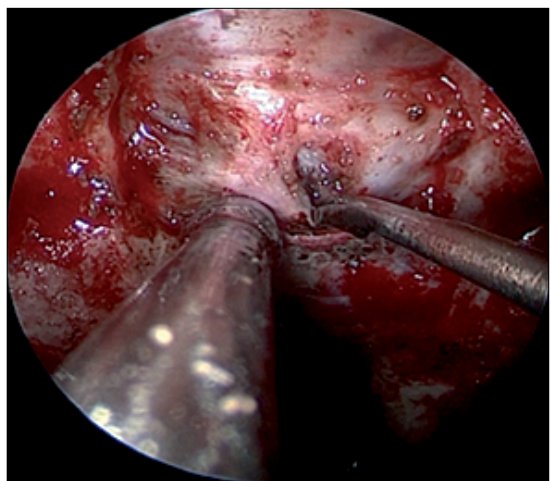

Figura 2. Apertura dural en el área infraquiasmática.

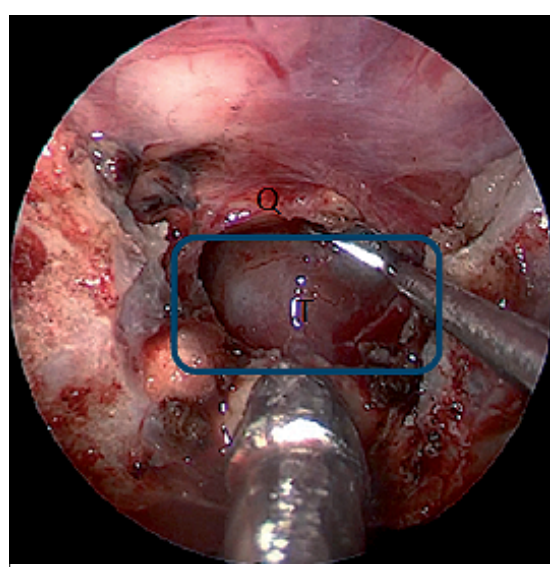

Figura 4. Identificación del corredor quirúrgico infraquiasmático.Q: Quiasma óptico, T: Tumor.

giomas retroinfundibulares es necesaria la osteotomía total de la silla turca para realizar un abordaje transdorsoselar con transposición de la glándula anterosuperior hacia el ángulo anterior del quiasma óptico (transposición de la glándula $)^{5}$. Es importante señalar que de los tres complejos anatómicos solamente el inferior es el que puede ser movilizado sin gran repercusión clínica, los demás son complejos fijos.

Posteriormente, se realiza la disección y exéresis tumoral siguiendo los principios generales de debulking tumoral y disección de la cápsula con hidrodisección e instrumentos filosos. Cabe señalar que la disección cuidadosa de la cápsula tumoral del hipotálamo subyacente es determinante en la evolución clínica posoperatoria de forma exponencial.

Una vez realizada la exéresis tumoral 
por vía subquiasmática, se debe realizar una exploración supraquiasmática, con el objetivo de alcanzar fragmentos tumorales de la cúpula tumoral que se encuentren en una posición alta en el tercer ventrículo (Figura 5).

Posteriormente, se debe realizar un lavado amplia con solución salina a $37^{\circ} \mathrm{C}$, recordar que esta es la temperatura del punto de ajuste hipotalámico, evitando así trastornos de la temperatura precoz. La inspección infraquiasmática nos muestra las arterias cerebrales anteriores, el top de la basilar, las arterias comunicantes posteriores en dirección a P1, así como el tercer ventrículo desde una perspectiva antero-inferior. Se observa además los agujeros de Monro y el plexo coroides (Figura 6).

Para evaluar el comportamiento clínico posoperatorio se utilizó la escala estándar de evaluación clínica para Craneofaringiomas (Elliot y colaboradores 2010), donde se tuvieron en cuenta: el estatus neurológico, el estatus visual, el estatus pituitario y el estatus hipotalámico ${ }^{1}$.

El Estatus Neurológico se clasificó en: Grado 1: no déficit ni convulsiones.

Grado 2: parálisis de nervio craneal y convulsiones controladas.

Grado 3: hemiparesia leve con deambulación independiente, convulsiones moderadamente controlada.

Grado 4: hemiparesia moderada y severa, abulia, letargo.

El Estatus Visual se clasificó en:

Grado 1: agudeza visual y campo visual normal.

Grado 2: Déficit leve de agudeza y campo visual.

Grado 3: ceguera unilateral, hemianopsia homónima o hemianopsia bitemporal.

Grado 4: ceguera bilateral.

El Estatus Pituitario se clasificó en:

Grado 1: función glandular normal.

Grado 2: leve disfunción pituitaria anterior (1 o 2 suplementos hormonales).

Grado 3: diabetes insípida con o sin disfunción leve anterior.

Grado 4: diabetes insípida con panhipopituitarismo.

El Estatus Hipotalámico se clasificó en: Grado 1: función hipotalámica normal. Grado 2: obesidad sin trastornos psicológicos ni del comportamiento.

Grado 3: obesidad con hiperfagia y

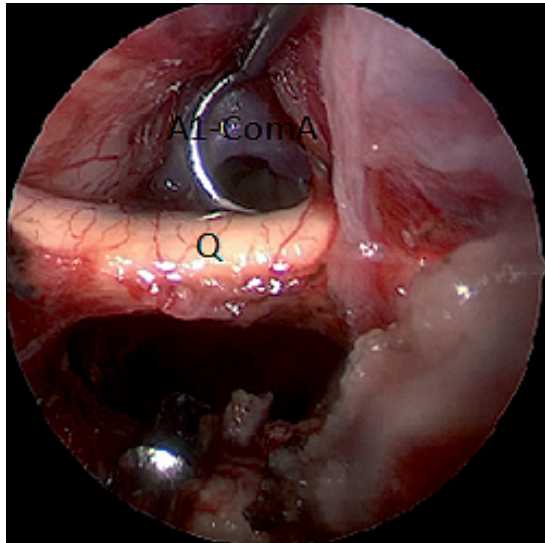

Figura 5. Exploración endoscópica supraquiasmática. Q: quiasma óptico, A1-ComA: Complejo cerebral anterior-comunicante anterior.

trastornos de memoria.

Grado 4: obesidad extrema, hiperfagia cambios de conducta con episodios de ira, trastornos de memoria, del ciclo vigilia-sueño y de la termorregulación.

Se determinaron las dimensiones del tumor y se calculó el volumen según la fórmula del elipsoide.

Volumen $\left(\mathrm{cm}^{3}\right)=\underline{0,5(\text { largo } x \text { ancho } x \text { alto en } \mathrm{mm})}$ 1000

Para evaluar el grado de Resección Tumoral se realizó la Tomografía Axial Computarizada a las 24 horas de operados y Resonancia Magnética Nuclear a los 3 meses, se procedió a clasificar en:

- Resección total: 100\%.

Resección subtotal: $75-99 \%$.

Resección parcial: $50-74 \%$.

Biopsia: Menos del 50\%.

\section{Resultados y discusión}

Fueron intervenidos quirúrgicamente 40 pacientes con Craneofaringiomas, 30 de ellos del tipo adamantinomatoso y 10 papilares (Tabla 1 ). En la literatura se evidencia que la variante adamantinomatosa está presente en más de $95 \%$ de los casos pediátricos y la variante escamosa-papilar es casi exclusivamente vista en adultos y representa cerca del $30 \%$ en esta población ${ }^{5,6,7}$. El cuanto al estatus visual se aprecia que en la evaluación preoperatoria, predominaba el grado 3 , sin embargo, después de la cirugía se observó un mejoría hacia los grados 1 y 2 . En varios trabajos y publicaciones anteriores

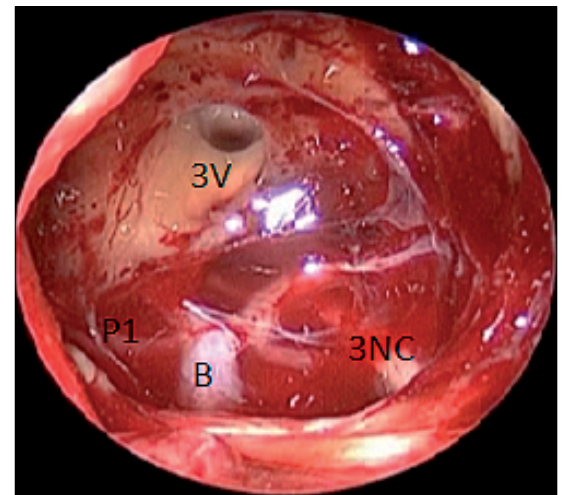

Figura 6. Inspección endoscópica infraquiasmática. B: Basilar; P1: segmento P1 de la arteria cerebral posterior; $3 \mathrm{NC}$ : tercer nervio craneal; $3 \mathrm{~V}$ : tercer ventrículo.

se ha planteado una tendencia simi$\operatorname{lar}^{7-11}$, el acceso directo a la lesión y la posibilidad de la descompresión de la vía visual desde la perspectiva anteroinferior con una identificación y disección clara y segura de las estructuras son beneficios particulares del abordaje endoscópico. Las manifestaciones visuales en los pacientes con Craneofaringiomas derivan del efecto compresivo sobre el quiasma óptico, desplazándolo hacia arriba y delante ${ }^{5,12,13}$.

En cuanto a la función pituitaria no se observó variación entre los estatus preoperatorio y posoperatorio, y en el caso del estatus hipotalámico no se observó mejoría del mismo después de la cirugía y en 6 pacientes existió un empeoramiento del mismo. El efecto invasivo del craneofaringioma a través de la liberación de enzimas lisosomales proteolíticas denominadas catepsinas $^{4,12}$, produce un daño irreversible en las estructuras adyacentes, sobre todo de la glándula hipófisis y del hipotálamo, lo cual se expresa como un hipopituitarismo progresivo, y las manifestaciones clínicas de daño hipotalámico como cambios de conducta, diabetes insípida, hiperfagia, trastornos de la memoria, y en último caso trastorno severo en la regulación del equilibrio hidro-elecrolítico con pérdida del mecanismo de la sed así como cambios en la regulación de la temperatura corporal. Los síntomas que se derivan del efecto compresivo de la lesión tumoral, pueden mejorar después de la cirugía si no tienen un carácter crónico, por ejemplo, la hemianopsia heterónima bitemporal secundaria a la compresión del quiasma óptico, sin embargo, los 
Tabla 1.

Resultados del Abordaje Endonasal Endoscópico Extendido en los pacientes con Craneofaringiomas

\begin{tabular}{|l|l|}
\hline Cantidad de pacientes & $\mathbf{n}=\mathbf{4 0}$ \\
\hline Tipo de Craneofaringioma & Papilar (10); Adamantinomatoso (30) \\
\hline Estatus Visual & $\begin{array}{l}\text { Pre-op G- 1 (3); G- 2 (8); G- 3 (27); G- 4 (2) } \\
\text { Pos-op G- 1 (18); G- 2 (16); G- 3 (6); G- 4 (0) }\end{array}$ \\
\hline Estatus Pituitario & $\begin{array}{l}\text { Pre-op G- 1 (3); G- 2 (12); G- 3 (10); G- } 4 \text { (15) } \\
\text { Pos-op G- 1 (4); G- 2 (10); G- 3 (10); G- 4 (16) }\end{array}$ \\
\hline Estatus Hipotalámico & $\begin{array}{l}\text { Pre-op G- 1 (7); G- 2 (20); G- 3 (11); G- } 4 \text { (2) } \\
\text { Pos-op G- 1 (7); G- 2 (18); G- 3 (7); G- 4 (8) }\end{array}$ \\
\hline Estatus Neurológico & $\begin{array}{l}\text { Pre-op G- 1 (27); G- 2 (7); G- 3 (6); G- } 4 \text { (0) } \\
\text { Pos-op G- 1 (28); G- 2 (6); G- 3 (5); G- } 4 \text { (1) }\end{array}$ \\
\hline Tipo de Abordaje Endonasal Endoscópico Extendido & TTTmS (21); TTTS (10); TTTDS (9) \\
\hline Grado de Resección Tumoral & Total (37); Subtotal (3), Parcial (0), Biopsia(0) \\
\hline Complicaciones & $\begin{array}{l}\text { Diabetes insípida (24), fístula de LCR (2), meningitis (1), } \\
\text { fallecidos (3) }\end{array}$ \\
\hline $\begin{array}{l}\text { Leyenda: Preoperatorio (Pre-op); Posoperatorio (Pos-op); Grado (G); Transplanum-transtuberculum-transmedio selar } \\
\text { (TTTmS); Transplanum-transtuberculum-transelar (TTTS); Transplanum-transtuberculum-transdorsoselar (TTDS); Líquido } \\
\text { cefalorraquídeo (LCR). }\end{array}$ \\
\hline
\end{tabular}

efectos secundarios a la acción invasiva del tumor sobre las estructuras adyacentes, generalmente no mejoran después de la cirugía, en estos casos al retirar la parte sólida tumoral se lograría una estabilización de los síntomas, estos elementos apoyan la realización de una cirugía bien precoz, antes que los efectos invasivos del tumor alcancen grados considerables, pues mientras exista el componente sólido estamos en presencia de una enfermedad tumoral con efectos neuroendocrinos en progresión irreversible ${ }^{12}$. A todo esto hay que añadirle el daño que se produce en el eje hipotálamo-hipofisario durante la disección en el acto quirúrgico. En la evaluación del estatus neurológico no se apreció variación significativa posoperatoria (Tabla 1).

En cuanto a la resección tumoral se muestra que la resección fue total en 37 pacientes $(92,5 \%)$ y subtotal en 3 pacientes $(7,5)$, no existieron resecciones parciales ni simples biopsias. La remoción quirúrgica de un Craneofaringioma constituye un desafío debido a su localización profunda y su íntima relación con estructuras neuro-vasculares críticas. La resección completa ha demostrado proveer un gran beneficio en cuanto a la supervivencia, pero a menudo a expensa de funciones neurológicas, oftalmológicas y endocrinológicas $^{13,14,15}$. Con el uso del abordaje transesfenoidal se proporciona una visión directa desde la perspectiva central e inferior de toda la región supraselar sin retracción de estructuras neuro-vasculares $^{4,5}$, inicialmente este abordaje fue reservado para Craneofaringiomas principalmente sub-diafragmáticos con silla turca aumentada de tamaño, sin embargo, con el surgimiento del abordaje extendido y del corredor Transplanum- Transtubérculum acompañado de la magnificación endoscópica se hacen accesibles Craneofaringiomas que ocupan completamente el espacio supraselar y que presentan anatómicamente silla turca normal ${ }^{13,14,15,16}$.

Otras series usando también el abordaje endoscópico han reportado porcientos de resección total de $80 \%$, subtotal $17 \%$ y parcial $3 \%{ }^{17,18}$. Otros autores reportan con el abordaje transesfenoidal microquirúrgico entre un $6,9 \%$ y $90 \%$ de resección total sin embargo, hay autores que plantean que la magnificación y visualización del objetivo quirúrgico se ven limitadas por el uso del especu$\mathrm{IO}^{5,19-22}$. Cuando son usados los abordajes transcraneales como el Transbasal Subfrontal, el Interhemisférico, Pterional u Orbito-zigomático el corredor quirúrgico convencional interóptico u óptico-carotideo no permite una exposición adecuada de toda la lesión y en regiones como infra y retroquiasmática no se logra una visualización óptima ${ }^{14,23,24}$. Al-Mefty y Hakuba ${ }^{12,25,26}$, han definido el Abordaje Petrosal Posterior para Craneofaringiomas que crecen a la porción retroquiasmática, sin embargo, este abordaje tiene la desventaja de la retracción prolongada del lóbulo temporal lo que potencia la lesión de la vena de Labbé y la pérdida de la orientación de la línea media por la proyección lateral. Autores como Yasargil han reportado un $90 \%$ de resección total del tumor, con un $9 \%$ de mortalidad intraoperatoria y postoperatoria temprana y un $16,7 \%$ de mortalidad general ${ }^{27}$. Aunque se trata de tumores con gran índice de recurrencia ${ }^{14,28,29} \mathrm{el}$ mayor índice recrecimiento de residuos tumorales y morbilidad consecuente se observa en las resecciones parciales o limitadas ${ }^{28,29}$ mientras las resecciones radicales, aunque con más riesgo, presentan un mayor intervalo libre de la enfermedad ${ }^{30,31,32}$

La complicación más frecuente fue la Diabetes Insípida (Tabla 1), presente en 24 pacientes $(60 \%)$, la cual se corresponde con la íntima relación que presentan estos tumores con el tallo hipofisario lo cual no siempre hace posible su disección exitosa. Otras series reportan esta complicación entre un $23 \%$ y un $69 \%$ con la cirugía transesfenoidal ${ }^{8,9,32}$. Otras complicaciones como las fístulas de líquido cefalorraquídeo y la meningitis se reportaron aunque en menor cuantía. En todos nuestros casos se realizó el cierre de la base craneal con un colgajo pediculado y 
vascularizado de mucosa septal lo cual pensamos que fue determinante en estos resultados, así como la profilaxis quirúrgica con cefuroxima (750 mg cada 8 horas) durante 5 días. La mortalidad quirúrgica es representada en un $7,5 \%$ (3 pacientes). En uno de estos pacientes se presentó un accidente quirúrgico durante la disección de la cápsula tumoral donde se lesionó la arteria cerebral posterior. Los restantes fueron por complicaciones médicas en el postoperatorio; uno por disfunción hipotalámica global y uno por trombo- embolismo pulmonar (TEP). Otros autores que tienen como principio una cirugía agresiva señalan un $4,1 \%$ de mortalidad $^{3}$.

\section{Conclusiones}

Los Craneofaringiomas son tumores que crecen en relación con el área regulatoria del equilibrio y estabilidad dinámica del medio interno así como del eje hormonal central, por tanto, el abordaje preciso, la disección meticu- losa por etapas y el respeto al hipotálamo y red vascular subyacente son determinantes en la evolución clínica posoperatoria. Los síntomas derivados del efecto compresivo del tumor mejoran después de la cirugía, sin embargo, los derivados del efecto invasivo del tumor no mejoran, pero se estabilizan después de la operación.

\section{Recibido: 01 de mayo de 2017} Aceptado: 22 de mayo de 2017

\section{Referencias}

1. Elliott RE, Sands SA, Strom RG, Wisoff JH. Craniopharyngioma. Clinical Status Scale: astandardized metric of preoperative function and posttreatment outcome. Neurosurg Focus. 2010; 28: E2.

2. Kassam AB, Gardner PA, Snyderman CH, Carrau RL, Mintz AH, Prevedello DM. Expanded endonasal approach, a fully endoscopic transnasal approach for the resection of midline suprasellar craniopharyngiomas: a new classifiation based on the infundibulum. $J$ Neurosurg. 2008; 108: 715-728.

3. Puget S, Garnett M, Wray A, et al. Pediatric craniopharyngiomas: classification and treatment according to the degree of hypothalamic involvement. J Neurosurg 2007; 106: 3-12.

4. Benedetta Ludovica Pettorini, MD, Paolo Frassanito MD, Massimo Caldarell I MD. Molecular pathogenesis of craniopharyngioma: switching from a surgical approach to a biological one. Neurosurg Focus 28 (4):E1, 2010.

5. King AJ, BlacK M. Youmans Neurological Surgery. Craniopharyngioma. Chapter 135. 2012.

6. Crotty TB, Scheithauer BW, Young WF Jr, Davis DH, Shaw EG, Miller GM, et al. Papillary craniopharyngioma: a clinicopathological study of 48 cases. J Neurosurg. 1995; 83(2): 206-214.

7. Fernández-Miranda JC, Gardner PA, Snyderman CH, Devaney KO, Strojan P, Suárez C, Genden EM, Rinaldo A, Ferlito A. Craniopharyngioma: A pathologic, clinical, and surgical review. Head Neck. 2011 May 16. doi: 10.1002/hed.21771.

8. Páramo Fernández C, Alfonso A. Guía Clínica del Diagnóstico y Tratamiento de Craneofaringioma y otras lesiones Paraselares. Revista de Endocrinología. 2007; 54(1).13-22. Barcelona. España.

9. Laws ER Jr. Transsphenoidal removal of craniopharyngioma. Pediatr Neurosurg 1994; 21(Suppl 1): 57-63.

10. Buslei R, Nolde M, Hofmann B, et al. Common mutations of beta-catenin in adamantinomatous craniopharyngiomas but not in other tumours originating from the sellar region. Acta Neuropathol 2005;109: 589-597.

11. Di Rocco C, Caldarelli M, Tamburrini G, Massini L (2006) Surgical management of craniopharyngiomas - experience with a pediatric series. J Pediatr Endocrinol Metab 19(Suppl 1): 355-366.

12. López AO, Lobaina OM, Ortiz MM. Craneofaringiomas. Riesgos y desafíos del Abordaje Endonasal Endoscópico Extendido a la Base del Cráneo. Rev. Chil. Neurocirugía 40: 12-17, 2014

13. Yamada S, Fukuhara N, Oyama K, Takeshita A, Takeuchi Y, Ito J, Inoshita N. Surgical outcome in 90 patients with craniopharyngioma: an evaluation of transsphenoidal surgery. World Neurosurg. 2010 Aug-Sep; 74(2-3): 320-330.

14. Frank G, Pasquini E, Doglietto F, Mazzatenta D, Sciarretta V, Farneti G, Calbucci F. The endoscopic extended transsphenoidal approach for craniopharyngiomas. Center of Surgery for Pituitary Tumours, Department of Neuroscience, Bellaria Hospital, Bologna, Italy. 2009 Jul:59(1 Suppl 1):ONS75-83; discussion ONS75-83.

15. Gonzalo L, Ullán J. Embriología. Ediciones Universidad de Navarra S. A. España. 1976.

16. Snell R. Embriología médica. $2^{\circ}$ edición. Nueva Editorial Interamericana. 1976. Madrid.

17. Cappabianca P, Esposito F, Cavallo LM, et al. Abordajes transnasales a la base de cráneo. In Gagliardi C, Fernández Molina G, Cuello LM. Técnicas actuales en neurocirugía endoscópica. 1르 ed. Ediciones de la Guadalupe. Buenos Aires. 2007: 269-288.

18. Moore Persaud. Embriología Clínica 7ํe edición. Editorial: Elsevier Science. Madrid, España. 2004.

19. Tena-Suck ML, Salinas-Lara C, Arce-Arellano RI, Rembao-Bojourquez D, Morales-Espinosa D, Sotelo J, Arrieta O (2006) Clinicopathological and immunohistological characteristics associated to recurrence/regrowth of craniopharyngiomas. Clin Neurol Neurosurg 22: 661-669.

20. Haupt R, Magnani C, Pavanello M, Caruso S, Dama E, Garre ML. Epidemiological aspects of craniopharyngioma. J Pediatr Endocrinol Metab. 2006; 19 Suppl 1: 289-293.

21. Karavitaki N, Brufani C, Warner JT, et al. Craniopharyngiomas in children and adults: systematic analysis of 121 cases with long-term follow-up. Clin Endocrinol (Oxf). 2005; 62: 397-409.

22. Van Effenterre R, Boch AL. Craniopharyngioma in adults and children: a study of 122 surgical cases. J Neurosurg 2002; 97 : 3-11.

23. Defoort-Dhellemmes S, Moritz F, Bouacha I, et al. Craniopharyngioma: ophthalmological aspects at diagnosis. J Pediatr Endocrinol Metab. 2006; 19(suppl 1): 321-324.

24. O'Gorman CS, Simoneau-Roy J, Pencharz P, MacFarlane J, MacLusky I, Narang I, et al. Sleep-disordered breathing is increased in obese adolescents with craniopharyngioma compared with obese controls. J Clin Endocrinol Metab.

25. Al-Mefty O, Ayoubi S, Kadri PA. The petrosal approach for the resection of retrochiasmatic craniopharyngiomas. Neurosurgery $2008 ; 62$ Suppl 2: ONS-331-6. 
26. Hakuba A, Nishimura S, Inoue Y. Transpetrosal-transtentorial approach and its application in the therapy of retrochiasmatic craniopharyngiomas. Surg Neurol 1985; 24: 405-441.

27. Yasargil MG, Curcic M, Kis M, et al. Total removal of craniopharyngiomas. Approaches and long-term results in 144 patients. J Neurosurg 1990; 73: 3-11.

28. Louis DN, Ohgaki H, Wiestler OD, Cavenee WK. (Eds). WHO Classification of Tumours of the Central Nervous System. IARC. Lyon 2007; 14: $238-241$.

29. lughetti L, Bruzzi P. Obesity and craniopharyngioma. Ital J Pediatr 2011; 37: 38.

30. Maira G, Anile C, Albanese A, Cabezas D, Pardi F, Vignati A. The role of transsphenoidal surgery in the treatment of craniopharyngiomas. J Neurosurg 2004; 100: 445- 451.

31. Oldfield EH. Transnasal endoscopic surgery for craniopharyngiomas. Neurosurg Focus. 2010; 28: E8a.

32. Morisako H, Goto T, Goto H. Aggressive surgery based on an anatomical subclassifiation of craniopharyngiomas. Neurosurg Focus 41 (6): E10, 2016.

\section{Correspondencia a:}

Dr. Marlon Ortiz M.

Neurocirujano. Hospital Hermanos Ameijeiras.

La Habana. Cuba.

marlonneuro@gmail.com 